\title{
Probabilistic Robustness Analysis of Uncertain LTV Systems in Linear Fractional Representation
}

\author{
Luca Evangelisti and Harald Pfifer
}

\begin{abstract}
This letter quantifies the effect of random model uncertainty on finite horizon linear time-varying (LTV) systems. Mean and standard deviation field are approximated with high accuracy and efficiency by a Hilbert space technique called polynomial chaos expansion (PCE). The deterministic expansion coefficients of the generalized Fourier series are determined via orthogonal projection, also known as Galerkin projection. We propose the projection of uncertain systems in linear fractional representation (LFR), which can have computational benefits. The technique is benchmarked on a two-link robotic manipulator.
\end{abstract}

Index Terms - Robust control, time-varying systems, uncertain systems

\section{INTRODUCTION}

$\mathbf{T}$ HIS letter considers the probabilistic robustness analysis of finite horizon linear time-varying (LTV) systems. Such systems frequently arise in engineering applications, e.g., robotic manipulators [1], space launch vehicles [2], or aircraft [3], [4]. The considered uncertainties are parametric with a given probability distribution, which allows using theory of probability and function spaces. As a robustness metric, the mean and standard deviation from the nominal (unperturbed) trajectory are considered. Despite well-known advantages, e.g., lower conservatism in contrast to a deterministic worst-case analysis, it should be appreciated that the introduction of probabilities does not simplify the problem.

Traditionally, sampling-based black-box methods such as Monte Carlo have been used to tackle the problem. These methods provide, in general, simple yet slowly convergent approximations. Hence, they are often not very computationally efficient. More recently, based on the first applications to (nonlinear) systems and control [5], [6]; polynomial chaos based methods have sparked interest in probabilistic robust control, e.g., for stochastic LQR [7], linear parameter-varying [8], or model predictive [9] control. The theory of polynomial chaos has been available for quite some time [10]. A brief

Manuscript received February 16, 2021; revised April 14, 2021; accepted April 29, 2021. Date of publication XX XX, 2021; date of current version XX XX, 2021. This work was not supported by any organization. (Corresponding author: Luca Evangelisti.)

L. Evangelisti is with the Institute of System Dynamics and Control, German Aerospace Center (DLR), 82234 Wessling, Germany (e-mail luca.evangelisti@dlr.de).

$\mathrm{H}$. Pfifer is with the Faculty of Mechanical Science and Engineering, Technische Universität Dresden, 01062 Dresden, Germany (e-mail: harald.pfifer@tu-dresden.de) overview of it is given in Section III. Effectively, it exploits the structure of mappings under uncertainty via orthogonal projection on polynomial basis functions. Theoretically, polynomial chaos expansions (PCEs) promise a superior convergence over sampling-based methods. Still, for many engineering problems, PCE is computationally expensive.

The chief contribution of this letter is to simplify the PCE of uncertain LTV systems by applying a linear fractional transformation (LFT). The LFT separates the deterministic and stochastic problem parts, yielding a distinct problem structure. In this structure, the stochastic part is always given by a linear mapping, i.e., the uncertainty block $\Delta$ from classical robust control. This significantly reduces the computational effort required for the PCE of the uncertain system, see Section IV. The PCE allows for an efficient extraction of the stochastic moments for a probabilistic robustness analysis of the system's response under random model uncertainty.

The effectiveness of the proposed approach is demonstrated on a two-link robotic manipulator benchmark in Section V. The benchmark compares both a conventional PCE-based approach without structure separation and the LFT-based approach proposed in this letter with classical sampling-based approaches.

\section{NOTATION}

The letter follows a standard notation as introduced, e.g., in [11]-[13]. Let $(\Omega, \mathscr{F})$ be a measurable space and $(\Omega, \mathscr{F}, \mu)$ a probability space. The set $\Omega$ is the sample space, equipped with $\sigma$-algebra $\mathscr{F}$ and probability measure $\mu$. We consider $\mathbb{R}$-valued random variables, i.e., measurable functions $f:(\Omega, \mathscr{F}) \mapsto(\mathbb{R}, \mathscr{G})$, with Borel $\sigma$-algebra $\mathscr{G}$. Denote $L^{2}(\Omega, \mu ; \mathbb{R})$ the Hilbert space w.r.t. the inner product $\langle f, g\rangle_{L^{2}}=\int_{\Omega} f(\omega) g(\omega) \mathrm{d} \mu(\omega)=\mathbb{E}[f g]$ containing all $\mathbb{R}$-valued random variables $f$ with finite induced $L^{2}$-norm $\|f\|_{L^{2}}=\sqrt{\langle f, f\rangle_{L^{2}}}<\infty$. In favor of highlighting the association with orthogonality, the expectation $\mathbb{E}[\cdot]$ w.r.t. $\mu$ is written as $\langle\cdot\rangle$ when appropriate, e.g., $\langle f, g\rangle_{L^{2}}=\langle f g\rangle$.

We study $\delta$ as a $\mathbb{R}^{n_{\delta}}$-valued random vector whose entries are made up of independent random variables. For $i=1, \ldots, n_{\delta}$, let $\left(\Omega_{i}, \mathscr{F}_{i}, \mu_{i}\right)$ be a collection of probability spaces. The product probability space $(\Omega, \mathscr{F}, \mu)$ is defined via $\Omega=\Omega_{1} \times \ldots \times \Omega_{n_{\delta}}$, $\mathscr{F}=\mathscr{F}_{1} \otimes \ldots \otimes \mathscr{F}_{n_{\delta}}$, and $\mu=\mu_{1} \otimes \ldots \otimes \mu_{n_{\delta}}$. Finally, assuming separability [11], the $L^{2}$ space over the product probability space is equivalent to the Hilbert space tensor product, i.e., $L^{2}\left(\Omega_{1} \times \ldots \times \Omega_{n_{\delta}}, \mu_{1} \otimes \ldots \otimes \mu_{n_{\delta}} ; \mathbb{R}\right)=\otimes_{i=1}^{n_{\delta}} L^{2}\left(\Omega_{i}, \mu_{i} ; \mathbb{R}\right)$. 


\section{Spectral Methods for UnCERTAinty QUANTIFICATION}

This section provides a brief overview of spectral methods for uncertainty quantification, along the lines of [11], [12]. For a proper introduction, the reader is referred to the literature. This letter considers uncertain dynamic systems modeled by $n$th order ordinary differential equations (ODEs) over a finite time horizon $t \in[0, T]$. Assume the ODE can be written as

$$
\begin{aligned}
& 0=f(\stackrel{(n)}{y}(t, \delta), \ldots, y(t, \delta), \stackrel{(m)}{u}(t), \ldots, u(t), t, \delta) \\
& y(t=0)=y_{0}
\end{aligned}
$$

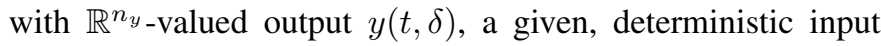
$u(t) \in \mathbb{R}^{n_{u}}, m \leq n$, and random model uncertainty $\delta \in$ $L^{2}(\Omega, \mu ; \mathbb{R})$. Generally, the initial condition $y_{0}$ can also be uncertain, but this is omitted for brevity. Further assume a unique solution to (1) exists for a given $u(t), y_{0}$, and for all values of $\delta$ occurring with non-zero probability. Uncertainty quantification studies the system's response surface viewed as a stochastic process, i.e., a function $y:[0, T] \times \Omega \mapsto \mathbb{R}^{n_{y}}$ such that each $y(t, \cdot)$ is a random vector for time $t \in[0, T]$.

Spectral methods ${ }^{1}$ characterize the stochastic process $y(t, \delta)$ in a mathematical fashion similar to Fourier series expansion for periodic time signals of finite energy. In essence, this breaks down to orthogonal decomposition, i.e., a series expansion

$$
y(t, \delta)=\sum_{k=0}^{\infty} y_{k}(t) \psi_{k}(\delta)
$$

with deterministic expansion coefficients $y_{k}$ and a suitable orthogonal basis $\left\{\psi_{k}\right\}_{k=0}^{\infty}$ of $L^{2}(\Omega, \mu ; \mathbb{R})$. Henceforth, function arguments will be omitted for notational convenience, when clear from the context. Note that the right-hand side of (2) assumes independence of the deterministic and stochastic function spaces [12]. The generalized Fourier coefficients $y_{k}$, also referred to as stochastic modes of $y$, are determined via the standard Hilbert space orthogonal projection

$$
y_{k}(t)=\frac{\left\langle y, \psi_{k}\right\rangle}{\left\langle\psi_{k}, \psi_{k}\right\rangle}=\frac{\int_{\Omega} y(t, \delta) \psi_{k}(\delta) \mathrm{d} \mu(\delta)}{\int_{\Omega} \psi_{k}^{2}(\delta) \mathrm{d} \mu(\delta)} .
$$

Note that function approximation by the above technique is analogous to the orthogonal projection of a point $p \in \mathbb{R}^{3}$ of the euclidean space onto a two-dimensional subspace, i.e., a plane.

Selecting multivariate orthogonal polynomials as a basis for $\otimes_{i=1}^{n_{\delta}} L^{2}\left(\Omega_{i}, \mu_{i} ; \mathbb{R}\right)$ results in what is known as generalized polynomial chaos ${ }^{2}$ (gPC) expansions. Note that such an orthogonal system of multivariate polynomials, which satisfies

$$
\begin{aligned}
\psi_{0} & =1 \\
\left\langle\psi_{k}, \psi_{l}\right\rangle & =\delta_{k l} \mathbb{E}\left[\psi_{k}^{2}\right] \quad \forall k, l \in \mathbb{N}_{0}
\end{aligned}
$$

with Kronecker delta $\delta_{k l}$, is yielded simply by taking products of the univariate orthogonal polynomials for $L^{2}\left(\Omega_{i}, \mu_{i} ; \mathbb{R}\right)$.

\footnotetext{
${ }^{1}$ Abstractly speaking, spectral methods can also be applied to uncertainty quantification of various other types of mathematical models, e.g., algebraic or partial differential equations.

${ }^{2}$ The term chaos has nothing to do with chaotic behavior of nonlinear dynamic systems. According to [11], it is more related to the study of stochastic processes and originates from the theory of Brownian motion [10].
}

The main motivation for selecting orthogonal polynomials is as follows. In practice, due to finite computational resources, the infinite series expansion (2) needs to be truncated to

$$
K+1=\frac{\left(n_{\delta}+d\right) !}{n_{\delta} ! d !}=\left(\begin{array}{c}
n_{\delta}+d \\
d
\end{array}\right)
$$

terms, i.e., a finite orthogonal basis consisting of multivariate polynomials with maximum total degree $d$. Thus, $y \approx \hat{y}=$ $\sum_{k=0}^{K} y_{k} \psi_{k}$, and there will be a truncation error orthogonal to $\operatorname{span}\left\{\psi_{0}, \ldots, \psi_{K}\right\}$, which is known to converge in the mean square sense [14]

$$
\lim _{K \rightarrow \infty}\|y-\hat{y}\|_{L^{2}}=0 .
$$

Furthermore, apart from the above limit, function approximation by orthogonal polynomial expansions improves exponentially as the smoothness of $y$ increases. This appealing property is known as spectral convergence. In order to elaborate, the notion of a Sobolov space needs to be defined. Denote $H^{k}(\Omega, \mu ; \mathbb{R})$ the Sobolov space consisting of all random variables $f$ such that all $j=0, \ldots, k$ (weak) derivatives $\frac{\mathrm{d}^{j} f}{\mathrm{~d} \omega^{j}} \in L^{2}(\Omega, \mu ; \mathbb{R})$. The Sobolev inner product and norm are $\langle f, g\rangle_{H^{k}}=\sum_{j=0}^{k}\left\langle\frac{\mathrm{d}^{j} f}{\mathrm{~d} \omega^{j}}, \frac{\mathrm{d}^{j} g}{\mathrm{~d} \omega^{j}}\right\rangle_{L^{2}}$ and $\|f\|_{H^{k}}=\sqrt{\langle f, f\rangle_{H^{k}}}$. Now the spectral convergence theorem for Legendre polynomials can be stated, which are the orthogonal polynomials for the uniform measure. A similar result can be shown for classical orthogonal polynomials, see [11].

Theorem 1 (Spectral convergence of Legendre polynomial expansions [11]). For all $f \in H^{k}(\Omega, \mu ; \mathbb{R})$, there exists a constant $C_{k} \geq 0$ that may depend upon $k$ but is independent of $d$ and $f$ such that

$$
\left\|f-\Pi_{d} f\right\|_{L^{2}}=\left\|f-\sum_{i=0}^{d} \frac{\left\langle f, \psi_{i}\right\rangle}{\left\langle\psi_{i}^{2}\right\rangle} \psi_{i}\right\|_{L^{2}} \leq C_{k} d^{-k}\|f\|_{H^{k}}
$$

where $\psi_{i}$ are orthogonal Legendre polynomials for $\mu=1 / 2$ on $\Omega=[-1,1]$

Thus, regularity of the function to be approximated increases the convergence rate in mean square. On the other hand, discontinuities can degrade convergence due to Gibbstype phenomena, see [12].

In addition to promising convergence properties, orthogonality of the PCE allows to derive explicit expressions for the statistical moments. For a stochastic process $y(t, \delta)$, this implies, e.g., mean field

$$
\mathbb{E}[y(t, \delta)]=\left\langle\psi_{0}, \sum_{k=0}^{\infty} y_{k}(t) \psi_{k}\right\rangle=y_{0}(t),
$$

covariance

$$
\begin{aligned}
C\left(t, t^{\prime}\right) & =\mathbb{E}\left[\left(y(t, \delta)-y_{0}(t)\right)\left(y\left(t^{\prime}, \delta\right)-y_{0}\left(t^{\prime}\right)\right)^{T}\right] \\
& =\left\langle\sum_{k=1}^{\infty} y_{k}(t) \psi_{k} \sum_{l=1}^{\infty} y_{l}\left(t^{\prime}\right)^{T} \psi_{l}\right\rangle \\
& =\sum_{k=1}^{\infty} y_{k}(t) y_{k}\left(t^{\prime}\right)^{T}\left\langle\psi_{k}^{2}\right\rangle,
\end{aligned}
$$


and variance

$$
\mathbb{V}[y(t, \delta)]=\sum_{k=1}^{\infty} y_{k}(t) y_{k}(t)^{T}\left\langle\psi_{k}^{2}\right\rangle .
$$

There are generally two approaches to determine the stochastic modes (3): intrusive and non-intrusive methods. Non-intrusive approaches treat the model (1) as a black-box and numerically compute the integral $\left\langle y, \psi_{k}\right\rangle$ using realizations of $\delta$, e.g., via deterministic Gaussian quadrature, Monte Carlo (MC) or quasi-MC integration. In contrast, intrusive methods rely on the Galerkin approach to project the model equations on the orthogonal basis spanned by $\psi_{k}$, yielding a $(K+1)$-times expanded system of generally coupled equations for the stochastic modes $y_{k}$. The latter approach is pursued in this letter.

\section{Polynomial Chaos Expansion of Uncertain LTV SYSTEMS}

\section{A. Galerkin Projection of Uncertain LTV Systems in Descriptor Form}

For $t \in[0, T]$, consider an uncertain LTV system in descriptor (implicit) form

$$
\begin{aligned}
E(t, \delta) \dot{x}(t, \delta) & =A(t, \delta) x(t, \delta)+B(t, \delta) u(t) \\
y(t, \delta) & =C(t, \delta) x(t, \delta)+D(t, \delta) u(t) \\
x(t=0) & =x_{0}
\end{aligned}
$$

with $\mathbb{R}^{n_{x}}$-valued state vector $x(t, \delta)$, input $u(t) \in \mathbb{R}^{n_{u}}$,

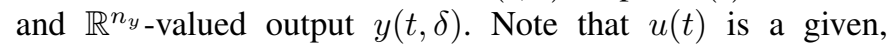
deterministic time signal, e.g., a certain control law. Assume that each $E, A, B, C, D$ is a known continuous mapping for all $[0, T] \times \Omega$ to a real matrix of appropriate dimension. Next, provided the mass matrix $E$ has full rank for all $[0, T] \times \Omega$, the aim is to apply Galerkin projection in order to determine the stochastic modes of the mappings $x(t, \delta)$ and $y(t, \delta)$.

Galerkin projection breaks down to inserting the gPC expansions of $\delta, x, y$, multiplying the equation by each $\psi_{k}$ for $k=0, \ldots, K$, and averaging, i.e., calculating the scalar product [11]. Thus, denoting

$$
\begin{array}{r}
x_{i}^{T}(t) \Psi(\delta):=\left[x_{i, 0}(t), \ldots, x_{i, K}(t)\right]\left[\begin{array}{c}
\psi_{0} \\
\psi_{1}(\delta) \\
\vdots \\
\psi_{K}(\delta)
\end{array}\right] \\
y_{i}^{T}(t) \Psi(\delta):=\left[y_{i, 0}(t), \ldots, y_{i, K}(t)\right]\left[\begin{array}{c}
\psi_{0} \\
\psi_{1}(\delta) \\
\vdots \\
\psi_{K}(\delta)
\end{array}\right]
\end{array}
$$

$$
\begin{aligned}
& \left\langle E(t, \cdot)\left[\begin{array}{c}
\dot{x}_{1}^{T}(t) \\
\vdots \\
\dot{x}_{n_{x}}^{T}(t)
\end{array}\right] \Psi \Psi^{T}\right\rangle=\left\langle A(t, \cdot)\left[\begin{array}{c}
x_{1}^{T}(t) \\
\vdots \\
x_{n_{x}}^{T}(t)
\end{array}\right] \Psi \Psi^{T}\right\rangle+\left\langle B(t, \cdot) u(t) \Psi^{T}\right\rangle, \quad\left\langle\left[\begin{array}{c}
x_{1}^{T}(0) \\
\vdots \\
x_{n_{x}}^{T}(0)
\end{array}\right] \Psi \Psi^{T}\right\rangle=\left\langle x_{0} \Psi^{T}\right\rangle \\
& \left\langle\left[\begin{array}{c}
y_{1}^{T}(t) \\
\vdots \\
y_{n_{y}}^{T}(t)
\end{array}\right] \Psi \Psi^{T}\right\rangle=\left\langle C(t, \cdot)\left[\begin{array}{c}
x_{1}^{T}(t) \\
\vdots \\
x_{n_{x}}^{T}(t)
\end{array}\right] \Psi \Psi^{T}\right\rangle+\left\langle D(t, \cdot) u(t) \Psi^{T}\right\rangle
\end{aligned}
$$

such that the transposition of (13) can be vectorized [using $\left.\operatorname{vec}(A B C)=\left(C^{T} \otimes A\right) \operatorname{vec}(B)\right]$ to

$$
\begin{aligned}
\mathcal{E}(t) \dot{X}(t) & =\mathcal{A}(t) X(t)+\mathcal{B}(t) u(t) \\
Y(t) & =\mathcal{C}(t) X(t)+\mathcal{D}(t) u(t)
\end{aligned}
$$

with deterministic initial condition $X(0)=x_{0} \otimes\langle\Psi\rangle$ and

$$
\begin{aligned}
& \mathcal{E}(t)=\left\langle E(t, \cdot) \otimes\left(\Psi \Psi^{T}\right)\right\rangle, \\
& \mathcal{A}(t)=\left\langle A(t, \cdot) \otimes\left(\Psi \Psi^{T}\right)\right\rangle, \mathcal{B}(t)=\langle B(t, \cdot) \otimes \Psi\rangle, \\
& \mathcal{C}(t)=\left(I_{n_{y}} \otimes\left\langle\Psi \Psi^{T}\right\rangle\right)^{-1}\left\langle C(t, \cdot) \otimes\left(\Psi \Psi^{T}\right)\right\rangle, \\
& \mathcal{D}(t)=\left(I_{n_{y}} \otimes\left\langle\Psi \Psi^{T}\right\rangle\right)^{-1}\langle D(t, \cdot) \otimes \Psi\rangle .
\end{aligned}
$$

Note that the gPC expansion of $\delta$ still needs to be inserted in (16), which has been omitted for better readability.

While (15) is generally an approximation to the stochastic dynamics (11) due to truncation, the projection integrals in (16) are assumed to be computable exactly, e.g., via Gaussian quadrature. However, based on the dependency on $\delta$, this can be a difficult task, see e.g. [12] for a detailed treatment of common nonlinearities. To illustrate, if the dependency on $\delta$ is polynomial with maximum total degree $d_{\delta}$, a multiplication tensor of order $d_{\delta}+2$ needs to be computed in order to calculate the inner products. In general, these multiplication tensors will have $(K+1)^{d_{\delta}+2}$ entries [11]. Note that multiplying the state equation in (11) by $E^{-1}(t, \delta)$ in order to obtain an explicit system representation complicates the projection integrals.

\section{B. Galerkin Projection of Uncertain LTV Systems in Linear Fractional Representation}

Instead, if the uncertain system (11) can be written as a linear fractional transformation - which is always possible if $\delta$ enters rationally [16] - Galerkin projection can be applied to the linear fractional representation (LFR) of the system. As depicted in Fig. 1, assume (11) can be transformed into the interconnection of a nominal system $G$

$$
\left[\begin{array}{c}
\dot{x}(t, \delta) \\
v(t, \delta) \\
y(t, \delta)
\end{array}\right]=\left[\begin{array}{ccc}
A_{G}(t) & B_{w}(t) & B_{u}(t) \\
C_{v}(t) & D_{v w}(t) & D_{v u}(t) \\
C_{y}(t) & D_{y w}(t) & D_{y u}(t)
\end{array}\right]\left[\begin{array}{c}
x(t, \delta) \\
w(t, \delta) \\
u(t)
\end{array}\right]
$$


with an isolated uncertain part

$$
w(t, \delta)=\Delta(\delta) v(t, \delta) .
$$

Analogous to robust control theory [16], $\Delta(\delta)$ denotes a random diagonal matrix

$$
\Delta(\delta)=\left[\begin{array}{ccc}
\delta_{1} I_{m_{1}} & & 0 \\
& \ddots & \\
0 & & \delta_{n_{\delta}} I_{m_{n_{\delta}}}
\end{array}\right]
$$

generated by repetition of each $\delta_{i}$ with multiplicity $m_{i}$ such that $n_{\Delta}=\sum_{i=1}^{n_{\delta}} m_{i}$. Note that writing the system in explicit form causes no extra difficulties (in contrast to the previous section), since the inverse and multiplication of LFTs are straightforward to compute [16].

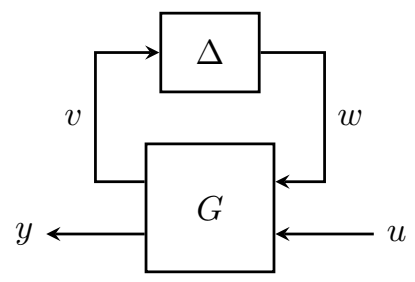

Fig. 1. Linear fractional representation of an uncertain LTV system

In addition to (14), denote

$$
\begin{aligned}
W(t) & =\left[w_{1}^{T}(t), \ldots, w_{n_{\Delta}}^{T}(t)\right]^{T} \in \mathbb{R}^{(K+1) n_{\Delta}} \\
V(t) & =\left[v_{1}^{T}(t), \ldots, v_{n_{\Delta}}^{T}(t)\right]^{T} \in \mathbb{R}^{(K+1) n_{\Delta}}
\end{aligned}
$$

the expansion coefficients of $w$ and $v$. Since the nominal system (17), i.e., $G$ in Fig. 1, is entirely deterministic, its stochastic modes are decoupled [as opposed to (15)]

$$
\left[\begin{array}{c}
\dot{X}(t) \\
V(t) \\
Y(t)
\end{array}\right]=\left[\begin{array}{ccc}
\mathcal{A}_{G}(t) & \mathcal{B}_{w}(t) & \mathcal{B}_{u}(t) \\
\mathcal{C}_{v}(t) & \mathcal{D}_{v w}(t) & \mathcal{D}_{v u}(t) \\
\mathcal{C}_{y}(t) & \mathcal{D}_{y w}(t) & \mathcal{D}_{y u}(t)
\end{array}\right]\left[\begin{array}{c}
X(t) \\
W(t) \\
u(t)
\end{array}\right]
$$

with

$$
\begin{aligned}
\mathcal{A}_{G}(t) & =A_{G}(t) \otimes I_{K+1} & \mathcal{B}_{w}(t) & =B_{w}(t) \otimes I_{K+1} \\
\mathcal{C}_{v}(t) & =C_{v}(t) \otimes I_{K+1} & \mathcal{D}_{v w}(t) & =D_{v w}(t) \otimes I_{K+1} \\
\mathcal{C}_{y}(t) & =C_{y}(t) \otimes I_{K+1} & & D_{y w}(t)=D_{y w}(t) \otimes I_{K+1}
\end{aligned}
$$

and

$$
\begin{aligned}
\mathcal{B}_{u}(t) & =\left(I_{n_{x}} \otimes\left\langle\Psi \Psi^{T}\right\rangle\right)^{-1}\left(B_{u}(t) \otimes\langle\Psi\rangle\right)=B_{u}(t) \otimes e_{1} \\
\mathcal{D}_{v u}(t) & =\left(I_{n_{\Delta}} \otimes\left\langle\Psi \Psi^{T}\right\rangle\right)^{-1}\left(D_{v u}(t) \otimes\langle\Psi\rangle\right)=D_{v u}(t) \otimes e_{1} \\
\mathcal{D}_{y u}(t) & =\left(I_{n_{y}} \otimes\left\langle\Psi \Psi^{T}\right\rangle\right)^{-1}\left(D_{y u}(t) \otimes\langle\Psi\rangle\right)=D_{y u}(t) \otimes e_{1} .
\end{aligned}
$$

In fact, these are sparse simple matrices, where $\langle\Psi\rangle=e_{1}$ is a $(K+1)$-dimensional unit vector $[1,0, \ldots, 0]^{T}$. The isolated uncertain part

$$
\begin{aligned}
W(t) & =\left(I_{n_{\Delta}} \otimes\left\langle\Psi \Psi^{T}\right\rangle\right)^{-1}\left\langle\Delta \otimes\left(\Psi \Psi^{T}\right)\right\rangle V(t) \\
& =: \Delta_{\Pi} V(t)
\end{aligned}
$$

is coupled solely via a third-order multiplication tensor [12] regardless of how $\delta$ originally enters (11), since $\Delta(\delta)$ is an affine mapping. The special structure of the Galerkin projection $\Delta_{\Pi}$ is indicated in (25). The decoupling and simplicity of Galerkin projection applied to uncertain systems in linear fractional representation stands out compared to the coupled system of equations derived in the previous section, where the treatment of rational parameter dependencies is computationally intensive in general.

\section{Application Example}

The effectiveness of the approach is analyzed in this section. Fig. 2 displays a two-link planar manipulator also studied in [17], with origin [1]. The rigid body equations of motion governing the mechanical system can be derived with the Lagrange formalism [1]

$$
\begin{aligned}
& {\left[\begin{array}{cc}
\alpha+2 \beta \cos \left(\theta_{2}\right) & \gamma+\beta \cos \left(\theta_{2}\right) \\
\gamma+\beta \cos \left(\theta_{2}\right) & \gamma
\end{array}\right]\left[\begin{array}{l}
\ddot{\theta}_{1} \\
\ddot{\theta}_{2}
\end{array}\right]+} \\
& {\left[\begin{array}{cc}
-\beta \sin \left(\theta_{2}\right) \dot{\theta}_{2} & -\beta \sin \left(\theta_{2}\right)\left(\dot{\theta}_{1}+\dot{\theta}_{2}\right) \\
\beta \sin \left(\theta_{2}\right) \dot{\theta}_{1} & 0
\end{array}\right]\left[\begin{array}{l}
\dot{\theta}_{1} \\
\dot{\theta}_{2}
\end{array}\right]=\left[\begin{array}{l}
\tau_{1} \\
\tau_{2}
\end{array}\right]}
\end{aligned}
$$

where the first term corresponds to the inertial forces, the second term denotes the Coriolis and centrifugal forces, and $\tau=\left[\tau_{1}, \tau_{2}\right]^{T}$ is the vector of applied torques. The robot's mass parameters are

$$
\begin{aligned}
\alpha & =I_{1}+I_{2}+m_{1} r_{1}^{2}+m_{2}\left(l_{1}^{2}+r_{2}^{2}\right) \\
\beta & =m_{2} l_{1} r_{2} \\
\gamma & =I_{2}+m_{2} r_{2}^{2}
\end{aligned}
$$

where each link has moment of inertia $I_{i}$, mass $m_{i}$, and length $l_{i}$. The distance between the $i$ th center of mass and joint is denoted $r_{i}$, see Fig. 2.

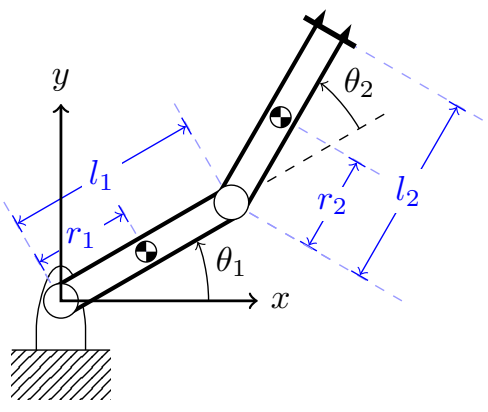

Fig. 2. Two-link planar manipulator

Deciding on a desired trajectory for the tool center point depicted in Fig. 3, a nominal reference trajectory $\dot{\Theta}^{*}(t), \Theta^{*}(t), \tau^{*}(t)$ for $t \in[0,5 \mathrm{~s}]$ and $\Theta=\left[\theta_{1}, \theta_{2}, \dot{\theta}_{1}, \dot{\theta}_{2}\right]^{T}$ is computed via inverse kinematics [1]. After linearization w.r.t.

$$
\Delta_{\Pi}=\left[\begin{array}{ccc}
I_{m_{1}} \otimes\left(\left\langle\Psi \Psi^{T}\right\rangle^{-1}\left\langle\delta_{1} \Psi \Psi^{T}\right\rangle\right) & & 0 \\
& \ddots & I_{m_{n_{\delta}}} \otimes\left(\left\langle\Psi \Psi^{T}\right\rangle^{-1}\left\langle\delta_{n_{\delta}} \Psi \Psi^{T}\right\rangle\right)
\end{array}\right]
$$


the reference trajectory, a linear quadratic regulator is designed for the nominal LTV system in order to minimize the quadratic cost

$$
J=x(T)^{T} F x(T)+\int_{0}^{T} x(t)^{T} Q x(t)+u(t)^{T} R u(t) \mathrm{d} t
$$

with $x=\Theta-\Theta^{*}, u=\tau-\tau^{*}, Q=\operatorname{diag}(10,1,10,1), R=I$, and $F=\operatorname{diag}(1,0.1,1,0.1)$. The optimal feedback control $u(t)=-R^{-1} B^{T} P(t) x(t)=K(t) x(t)$ is yielded by solving the associated Riccati differential equation [18].

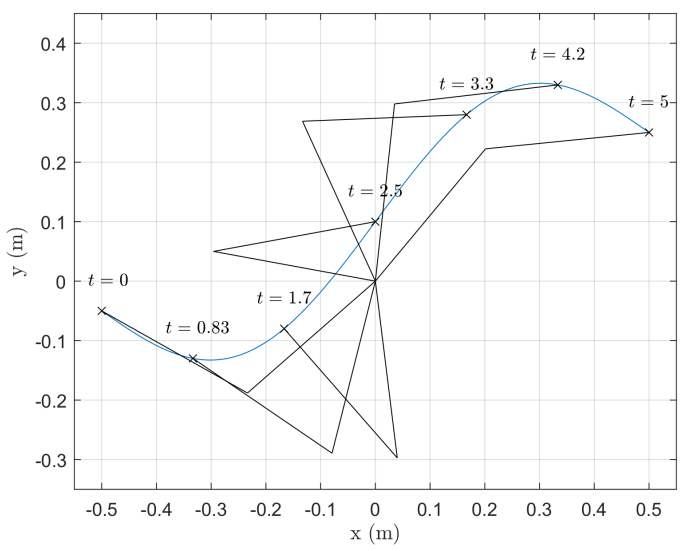

Fig. 3. Reference trajectory

The aim is to quantify the deviation of the closed-loop from the reference trajectory due to random model uncertainty in the robot's mass and inertial properties. Specifically, this is to be achieved by analysis of mean and standard deviation of the stochastic linear perturbations $x(t, \delta)$ about the reference trajectory if $\delta=\left[I_{1}, I_{2}, m_{1}, m_{2}, r_{1}, r_{2}\right]^{T}$ is modeled as a random vector, where each coordinate is distributed uniformly about its mean $\bar{\delta}_{i}$ with $\delta_{i} \sim U\left(0.5 \bar{\delta}_{i}, 1.5 \bar{\delta}_{i}\right)$. The robot's mean parameter values are $\bar{I}_{1}=0.09 \mathrm{~kg} \cdot \mathrm{m}^{2}, \bar{I}_{2}=0.06 \mathrm{~kg} \cdot \mathrm{m}^{2}$, $\bar{m}_{1}=3 \mathrm{~kg}, \bar{m}_{2}=2 \mathrm{~kg}, \bar{r}_{1}=\bar{r}_{2}=0.15 \mathrm{~m}$, and $l_{1}=l_{2}=0.3$ m.

Three types of algorithms are assessed neutrally for this task: direct Galerkin projection of the LTV system in descriptor form (DGK, see Section IV-A), Galerkin projection applied to the LFT system (LFTGK, see Section IV-B), and samplingbased estimates of mean

$$
\langle x(t, \delta)\rangle_{N}=\frac{1}{N} \sum_{i=1}^{N} x\left(t, \delta^{(i)}\right)
$$

and standard deviation

$$
\sigma\left(x_{i}(t, \delta)\right)=\sqrt{\frac{1}{N-1} \sum_{j=1}^{N}\left(x_{i}\left(t, \delta^{(j)}\right)-\left\langle x_{i}(t, \delta)\right\rangle_{N}\right)^{2}} .
$$

All computations are performed using the Julia programming language [19], on a standard desktop PC with $4 \mathrm{GHz}$ Intel i7 CPU and 16 GB RAM. For computing the Galerkin projections, the PolyChaos.jl toolbox [20] is applied. Time integrations are performed numerically by a fast order $2 / 3$ Rosenbrock solver with automatic differentiation for implicit systems and an order 5/4 Runge-Kutta method for explicit systems, each with relative tolerance $10^{-3}$ and absolute tolerance $10^{-6}[21]$.

Mean and standard deviation field of the LFTGK PCE with maximum total degree $d=3$ are plotted in Fig. 4. The number of coefficients (5) is $K+1=84$. The DGK approach yields identical results (within numerical tolerance).

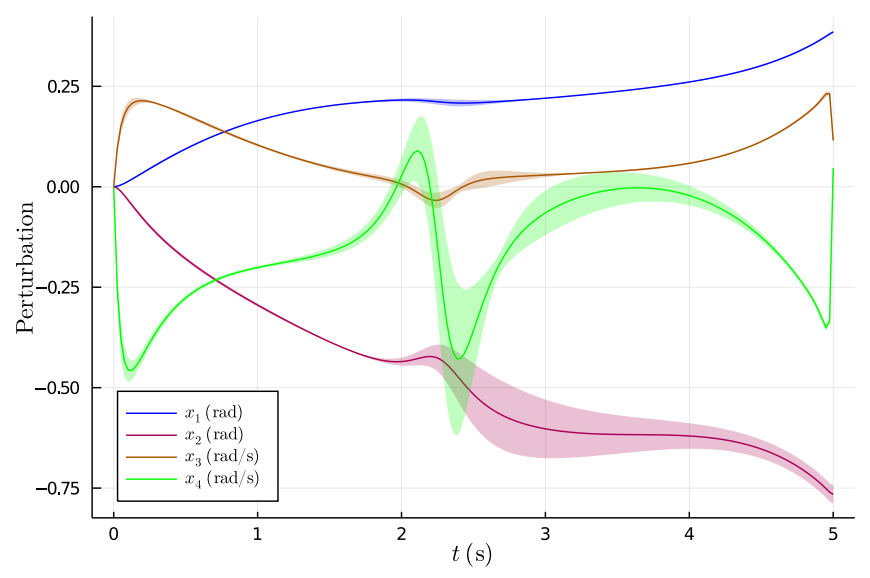

Fig. 4. Mean and standard deviation field of $\boldsymbol{d}=\mathbf{3}$ LFTGK PCE

The DGK approach from Section IV-A requires computation of multiplication tensors of order up to 5 in this example, and the calculation of the Galerkin projection takes about 2 $\mathrm{h}$. Although it is argued by practitioners that these are onetime reusable computations, this is in contrast to the LFTGK approach, which merely requires computation of a third-order multiplication tensor. The Galerkin projection of the uncertain LFT system has a decoupled special structure detailed in Section IV-B, such that the computation time is rather low (less than $1 \mathrm{~s}$ ).

Table I benchmarks a classical Monte Carlo sampling strategy with the PCE approaches from Section IV for $d \in$ $\{1,2,3\}$. The sample set dimension of the MC benchmark is scaled appropriately. As an unbiased reference solution, a Monte Carlo estimator with $N_{r e f}=10^{4}$ is used. Mean and standard deviation field error w.r.t. the reference are denoted by $e_{m}(t)$ and $e_{\sigma}(t)$, respectively, and $\|e(t)\|_{\mathcal{L}_{\infty}}=$ $\max _{i=1, \ldots, 4} \sup _{t}\left|e_{i}(t)\right|$ is the $\mathcal{L}_{\infty}$ signal norm. The last column shows the required computation time for solving the associated (system of) equations, excluding the calculation of inner products for the Galerkin projection. Note that solving the $d=3$ Galerkin projected system of equations takes (less than) $1 \mathrm{~min}$, whereas $N_{\text {ref }}=10^{4}$ samples require $37 \mathrm{~min}$ to solve. The decoupled structure of the LFT projection can also here be exploited advantageously in contrast to the coupled system of equations yielded by the DGK approach.

Fig. 5 performs a consistency check of the $d=3$ LFTGK PCE w.r.t. 3 sampling algorithms: MC, Latin Hypercube Sampling (LHS), and Sobol' sequence. Since the highest variability and largest approximation errors were observed in $x_{4}(t, \delta)$, the maximum (absolute) estimation error over time is analyzed in this coordinate. The empirical estimates of the first two statistical moments are compared with the $d=3$ 
TABLE I

BENCHMARK OF MC AND PCE

\begin{tabular}{clccc}
\hline & Dimension & $\left\|e_{m}(t)\right\|_{\mathcal{L}_{\infty}}$ & $\left\|e_{\sigma}(t)\right\|_{\mathcal{L}_{\infty}}$ & $\begin{array}{c}\text { Comp. } \\
\text { time }\end{array}$ \\
\hline DGK & $d=1$, & $1.4 \times 10^{-3}$ & $1.7 \times 10^{-2}$ & $1 \mathrm{~s}$ \\
LFTGK & $K+1=7$ & $2.3 \times 10^{-3}$ & $1.8 \times 10^{-2}$ & $1 \mathrm{~s}$ \\
MC & $N=7$ & $5.3 \times 10^{-2}$ & $1.0 \times 10^{-1}$ & $2 \mathrm{~s}$ \\
\hline DGK & $d=2$, & $1.5 \times 10^{-3}$ & $1.6 \times 10^{-3}$ & $11 \mathrm{~s}$ \\
LFTGK & $K+1=28$ & $2.0 \times 10^{-3}$ & $2.6 \times 10^{-3}$ & $6 \mathrm{~s}$ \\
MC & $N=50$ & $2.6 \times 10^{-2}$ & $1.5 \times 10^{-2}$ & $11 \mathrm{~s}$ \\
\hline DGK & $d=3$, & $1.5 \times 10^{-3}$ & $4.9 \times 10^{-4}$ & $60 \mathrm{~s}$ \\
LFTGK & $K+1=84$ & $1.9 \times 10^{-3}$ & $1.8 \times 10^{-3}$ & $16 \mathrm{~s}$ \\
MC & $N=250$ & $2.5 \times 10^{-3}$ & $1.9 \times 10^{-3}$ & $63 \mathrm{~s}$ \\
\hline & & & &
\end{tabular}

PCE as a surrogate for the exact solution. The fact that all sampling-based estimates converge (up to numerical accuracy) with roughly $N^{-1 / 2}$ - as expected [12] and indicated by the red line - to the PCE lends confidence to the approach.

In addition, the separate treatment of the deterministic and stochastic problem parts by the Galerkin approach is particularly advantageous if only the deterministic part changes. For example, if a new reference trajectory or controller is to be analyzed, the PCE can quickly provide an accurate updated estimate. This has to be contrasted with samplingbased or non-intrusive methods, which do not exploit the structure of the problem as the PCE does, and have to repeat the whole sampling campaign. The LFT Galerkin approach is able to exploit structure even further, since the LFR relieves the Galerkin projection of work during the separation of deterministic and uncertain parts.

\section{CONCLUSION}

This letter presented an approach for probabilistic robustness analysis of uncertain LTV systems, which exploits the structure yielded by a LFT before applying polynomial chaos theory. The benefits of the approach were illustrated on the example of a two-link robotic manipulator. Possible future applications lie, e.g., within the verification and validation process or online robustness analysis at reduced computational cost.

\section{ACKNOWLEDGMENT}

The authors gratefully acknowledge the thoughtful comments from and fruitful discussions with D. Oechsler from Technische Universität Dresden, Institute of Mathematical Stochastics.

\section{REFERENCES}

[1] R. Murray, Z. Li, and S. Sastry, A Mathematical Introduction to Robotic Manipulation. CRC Press, 1994.

[2] F. Biertümpfel, H. Pfifer, and S. Bennani, "Finite horizon worst case analysis of launch vehicles," in 21st IFAC Symp. Automatic Control in Aerospace, 2019, pp. 31-36.

[3] F. Biertümpfel and H. Pfifer, "Finite horizon touchdown analysis of autolanded aircraft under crosswind," in 4th IFAC Workshop on Linear Parameter Varying Systems, 2021, submitted for publication.

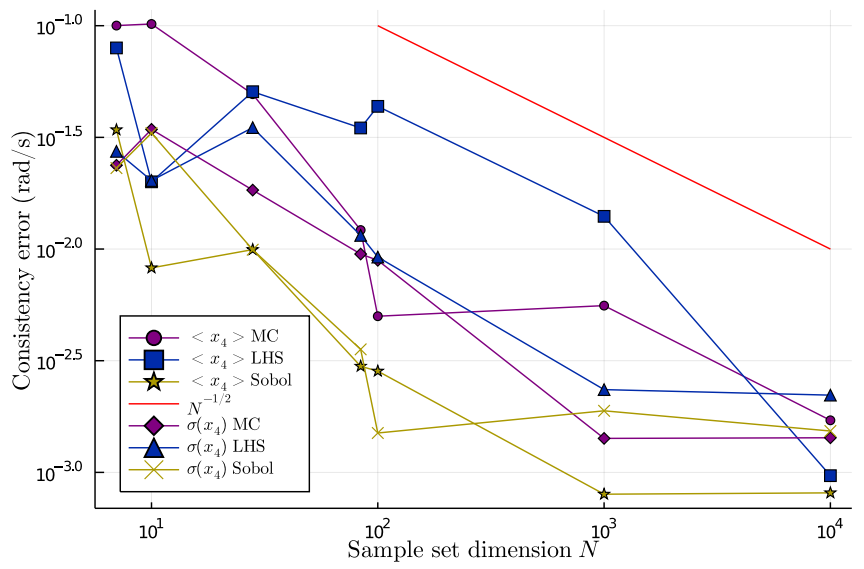

Fig. 5. Consistency check of $\boldsymbol{d}=\mathbf{3}$ LFTGK PCE surrogate

[4] A. Ianelli, P. Seiler, and A. Marcos, "Worst-case disturbances for time-varying systems with application to flexible aircraft," Journal of Guidance, Control, and Dynamics, vol. 42, pp. 1261-1271, 2019.

[5] F. Hover and M. Triantafyllou, "Application of polynomial chaos in stability and control," Automatica, vol. 42, pp. 789-795, 2006.

[6] Z. Nagy and R. Braatz, "Distributional uncertainty analysis using power series and polynomial chaos expansions," Journal of Process Control, vol. 17, pp. 229-240, 2007.

[7] J. Fisher and R. Bhattacharya, "Linear quadratic regulation of systems with stochastic parameter uncertainties," Automatica, vol. 45, pp. 28312841, 2009.

[8] R. Bhattacharya, "A polynomial chaos framework for designing linear parameter varying control systems," in Proc. Amer. Control Conf., 2015, pp. $409-414$.

[9] J. Paulson, A. Mesbah, S. Streif, R. Findeisen, and R. Braatz, "Fast stochastic model predictive control of high-dimensional systems," in Proc. 53rd IEEE Conf. Decis. and Control, 2014, pp. 2802-2809.

[10] N. Wiener, "The homogeneous chaos," American Journal of Mathematics, vol. 60, pp. 897-936, 1938.

[11] T. Sullivan, Introduction to Uncertainty Quantification. Springer, 2015.

[12] O. Le Maître and O. Knio, Spectral Methods for Uncertainty Quantification. Springer, 2010.

[13] M. Grigoriu, Stochastic Calculus. Birkhäuser, 2002.

[14] R. Cameron and W. Martin, "The orthogonal development of nonlinear functionals in series of fourier-hermite functionals," Annals of Mathematics, vol. 48, pp. 385-392, 1947.

[15] D. Shen, S. Lucia, Y. Wan, R. Findeisen, and R. Braatz, "Polynomial chaos-based $\mathcal{H}_{2}$-optimal static output feedback control of systems with probabilistic parametric uncertainties," in Proc. 20th IFAC World Congr., 2017, pp. 3536-3541.

[16] K. Zhou and J. Doyle, Essentials of Robust Control. Prentice Hall, 1998.

[17] P. Seiler, R. Moore, C. Meissen, M. Arcak, and A. Packard, "Finite horizon robustness analysis of ltv systems using integral quadratic constraints," Automatica, vol. 100, pp. 135-143, 2019.

[18] M. Green and D. Limebeer, Linear Robust Control. Prentice Hall, 1995.

[19] J. Bezanson, A. Edelman, S. Karpinski, and V. Shah, "Julia: A fresh approach to numerical computing," SIAM Review, vol. 59, pp. 65-98, 2017.

[20] T. Mühlpfordt, F. Zahn, V. Hagenmeyer, and T. Faulwasser, "Polychaos.jl - a julia package for polynomial chaos in systems and control," in Proc. 21st IFAC World Congr., 2020.

[21] C. Rackauckas and Q. Nie, "Differentialequations.jl-a performant and feature-rich ecosystem for solving differential equations in julia," Journal of Open Research Software, vol. 5, p. 15, 2017. 